\title{
Adaptive Sub-Aperture Integration for Wide-Angle Synthetic Aperture Radar
}

\author{
Shahzad Gishkori, David Wright, Liam Daniel, Aasim Shaikh, \\ Tareq Al-Naffouri, Marina Gashinova and Bernard Mulgrew
}

\begin{abstract}
In this paper, we present an adaptive sub-aperture integration method for wide-angle synthetic aperture radar (SAR) for improved imaging, with emphasis on short-to-medium range applications. In order to avoid full-aperture integration, traditional approaches use fixed-width sub-apertures, which may not conform to the persistence angle of the scatterers. Coherent integration gains over the aperture are possible if integration is carried out over the persistence angle of the scatterers, because integrating shorter than the persistence angle may spread the scattering response across multiple sub-apertures or, conversely, integrating more than the persistence angle may cause noise accumulation along with the useful signal. In this paper, we propose to use change-point detection methods to estimate the persistence widths of the scatterers, and consequently enhance the coherent integration gains, resulting in improved imaging. We compare our proposed methods with the standard integration approaches as well as a recently proposed adaptive integration approach. We provide qualitative and quantitative analyses to prove that our proposed methods outperform the existing approaches. We present experimental results on the real-data of our low-terahertz (THz) radar as well as a publicly available dataset to validate our claims.
\end{abstract}

Index Terms-THz radar, wide-angle SAR, sub-aperture integration, change-point detection

\section{INTRODUCTION}

Synthetic aperture radar (SAR) [2]-[4] can provide enhanced image quality in terms of spatial resolution by exploiting the diversity of viewing angles (see e.g., [5]-[8] and references therein). Consequently, a wide-angle SAR (i.e., where the radar measurements are collected over a large angular range) can significantly improve the image quality as it accumulates increased information of the target scene. Therefore, wide-angle SAR can envisage numerous applications, from a single sensor focusing on a target, to multiple sensors, e.g., on unmanned air vehicles (UAVs), working in

S. Gishkori, A. Shaikh and T. Al-Naffouri are with the Division of Computer, Electrical and Mathematical Sciences and Engineering (CEMSE), King Abdullah University of Science and Technology (KAUST), Kingdom of Saudi Arabia. Emails: \{shahzad.gishkori, mohammedaasim.shaikh, tareq.alnaffouri\}@kaust.edu.sa

B. Mulgrew and D. Wright (formerly) are with Institute for Digital Communications (IDCOM), The School of Engineering, The University of Edinburgh, United Kingdom. Emails: bernie.mulgrew@ed.ac.uk, dave_a_wright@yahoo.co.uk

L. Daniel and M. Gashinova are with Microwave Integrated System Laboratory (MISL), School of Electronic, Electrical and Systems Engineering, University of Birmingham, United Kingdom. Emails: \{1.y.daniel, m.s.gashinova\}@bham.ac.uk

This publication is based upon work supported in part by the King Abdullah University of Science and Technology (KAUST), Office of Sponsored Research (OSR), under Award No. ORA-CRG2021-4695.

Part of this paper was presented at 2020 IEEE Radar Conference [1]. concert, and even in bistatic radar scenarios. Due to their short wavelength, terahertz $(\mathrm{THz})$ radars (with/without SAR processing) have been gaining popularity for improved image resolution [9]-[11]. Recently, imaging capabilities of a low$\mathrm{THz}$ circular radar have been presented in [12], [13] for automotive context (i.e., for short-to-medium ranges), which also signifies the importance of wide-angle SAR for such civilian applications, and it forms the general theme of the present paper as well.

Theoretically, SAR (based on any synthesised aperture) is defined to be wide-angle if the aperture extent exceeds the angular sector (i.e., $2 \sin ^{-1}\left(B / 2 f_{0}\right)$, where $B$ is the bandwidth and $f_{0}$ is the carrier frequency), required for equal spatial bandwidth in range and cross-range [14], [15]. However, traditional SAR imaging techniques are based on the point scattering assumption, i.e., a target object can be modelled to be composed of a set of isotropic point scatterers whose reflection coefficients are independent of the angle of incidence. If this assumption is considered true, then, theoretically it is possible to obtain full circular SAR images with a spatial resolution of quarter of a wavelength over the Cartesian grid of the target scene [16]. In reality, this may not be possible. The point scattering assumption holds only for a small range of angles before it breaks down. For man-made objects with sharp corners and edges, the response is highly aspect dependent.

Generally, two broad integration approaches/methods are used to deal with anisotropic scatterers in wide-angle SAR, i.e., $i)$ the full-aperture (FA) methods and ii) the sub-aperture (SA) methods. The full-aperture processing methods model the scattering as a function of both space and azimuth across the full aperture [17]-[19]. Since the azimuth angle is explicitly adopted as an independent variable, the dimensionality of the image increases and requires some form of regularisation to recover the image from its samples [20]. Full aperture techniques are computationally expensive and are generally only applied to small regions of interest. Sub-aperture (or windowed) approaches [21]-[23] are the most commonly used approaches to deal with aspect dependent scatterers for wideangle data. Full aperture is divided into a series of smaller sub-apertures which span an angular range over which the point scattering assumption is valid and the reflector response is expected to be constant. This allows standard narrow-angle imaging methods to be applied within the sub-aperture [24], [25] to form a corresponding sub-image. An early example of this approach appeared in [26] and it was named 'aspectcoherent image formation'. The sub-aperture processing techniques, in contrast to full aperture, use a constant sub-aperture 
size and overlap distance, over the whole aperture. This is to ensure that the resolution of each sub-aperture is the same. The composite image of the target scene, per pixel, can then be formed by either combining the contributions of all subapertures non-coherently, i.e., sum of the absolute values, or by selecting the contribution of a sub-aperture with maximum absolute value. The composite image, in the latter case, has the interpretation of a generalised likelihood ratio test (GLRT) and is based on the assumption that the distribution of responses from a point at a given pixel is normally distributed [14]. The GLRT based composite image formation has received widespread acceptance from many researchers, e.g., [5], [6], [14], [15], [27].

Sub-aperture methods, with a constant aperture size, essentially assume that the scattering persistence angle (SPA), i.e, the angular range over which the scattering response of a scatterer is isotropic, is the same for all scatterers in the target scene. The resolution of a scatterer cannot be improved by integrating beyond the SPA of the scatterer, this simply adds noise to the image. Whereas, in case the sub-aperture is shorter than the SPA of a scatterer, the scattering response from the scatterer is spread across more than one sub-apertures which results in image blurring. However, a priori knowledge of the SPA of a scatterer is generally not available. Thus, fixing a constant size of the sub-aperture may not guarantee good image quality. One way to circumvent the problem of constant sub-aperture size is to select an adaptive sub-aperture size [28] corresponding to the SPA of the scatterers. Such an approach was used in [29] to improve image quality of a THz wide-angle SAR. The SPA of a scatterer was estimated by approximating the scattering energy of the scatterer via a Gaussian function. Finally, a common SPA was obtained for all the scatterers (assumingly based on the mean values). On the one hand, it is an approximate approach requiring certain assumptions especially in the case of overlapping SPAs. On the other hand, selecting a common SPA for all the scatterers essentially brings the technique closer to a constant sub-aperture approach. Therefore, only limited gains can be obtained via this technique, particularly in the shortto-medium-range $\mathrm{THz}$ sensing applications which exhibit large variations in aspect dependent scatterings.

In this paper, we propose an adaptive sub-aperture (AdSA) wide-angle $\mathrm{SAR}^{1}$ imaging approach where we estimate the SPA of the scatterers by employing a change-point detection strategy [32]-[36]. The change points basically capture the statistical variations, e.g., mean, root mean square (RMS) and standard deviation (STD), of the data segments. Thus, our proposed strategy offers a thorough and flexible search on estimating the SPA of a scatterer. Generally, the number of change points are pre-defined. However, an over-estimate of this number can also be utilised in our proposed method. In order to keep noise-only segments out, we utilise a threshold parameter which can be estimated from the data itself. Finally, coherent integration is applied over the estimated SPA of the scatterer, and a composite image is formed via

\footnotetext{
${ }^{1}$ Note, although we consider SAR imaging here, the proposed methodology is applicable to inverse SAR (ISAR) [30], [31] as well.
}

the GLRT method. Since the proposed method concerns an integration strategy over the aperture, it is independent of image reconstruction, per aperture sample. However, to make this point clearer, we consider images reconstructed by the classical back-projection method [4] as well as the recently proposed state-of-the-art super-resolution method [7] based on fused least absolute shrinkage and selection operator (fused LASSO) [37]. In order to implement our proposed strategy, we primarily utilise a dataset that we collected in our lab using a $300 \mathrm{GHz}$ radar in a circular scanning SAR (CiSSAR) mode [12]. The CiS-SAR mode has been found useful especially for short-to-medium range imaging applications (aimed by this paper as well). However, to make our proposed method data-independent, we also present some results on the publicly available dataset of a backhoe target [38]. In order to compare the performance of our proposed method, we present results of full-aperture coherent integration along with the constant sub-aperture coherent integration, as the reference standard approaches. In terms of comparison with an adaptive integration method, we also present some results by using the proposed method of [29]. These comparisons, via qualitative as well as quantitative analyses, prove the validity of our proposed method ${ }^{2}$.

Contributions. The following are the main contributions of this paper.

- We present an adaptive sub-aperture integration technique for circular (scanning) synthetic aperture radar for improved imaging.

- We employ the change-point detection method, which leverages statistical variations of the aperture data, in order to estimate the SPA of a scatterer.

- We compare our proposed methods with standard integration approaches as well as a recently proposed adaptive integration method.

- We provide experimental results on our $300 \mathrm{GHz}$ radar real-data, and a publicly available dataset, in order to prove the efficacy of our proposed methods, along with qualitative as well as quantitative analyses.

Organisation. Section II provides the system model and image reconstruction methods, Section III explains the concept of change-point detection, Section IV elaborates on the proposed adaptive integration technique, Section V demonstrates experimental results and Section VI gives the conclusions.

Notations. Matrices are in upper case bold while column vectors are in lower case bold, $(\cdot)^{T}$ denotes transpose, $[\mathbf{a}]_{i}$ is the $i$ th element of $\mathbf{a}$ and $[\mathbf{A}]_{i, j}$ is the $i j$ th element of $\mathbf{A}$, $\mathbf{a}$ is the estimate of $\mathbf{a}, \triangleq$ defines an entity, $|\mathcal{A}|$ denotes the cardinality of set $\mathcal{A}$, and the $\ell_{p}$-norm is denoted as $\|\mathbf{a}\|_{p}=\left(\sum_{i=1}^{N}\left|[\mathbf{a}]_{i}\right|^{p}\right)^{1 / p}$.

\footnotetext{
${ }^{2}$ Note, the proposed method was preliminarily presented in [1], along with qualitative assessment only. However, the present paper complements [1] by providing, i) a thorough treatment on the subject, ii) a detailed system model and theoretical foundations of the proposed method, iii) quantitative analyses of the performance results based on standard performance metrics, $i v)$ a comparison with a recently proposed approach on adaptive sub-aperture integration, and $v$ ) application of the proposed method on SAR images reconstructed through super-resolution.
} 


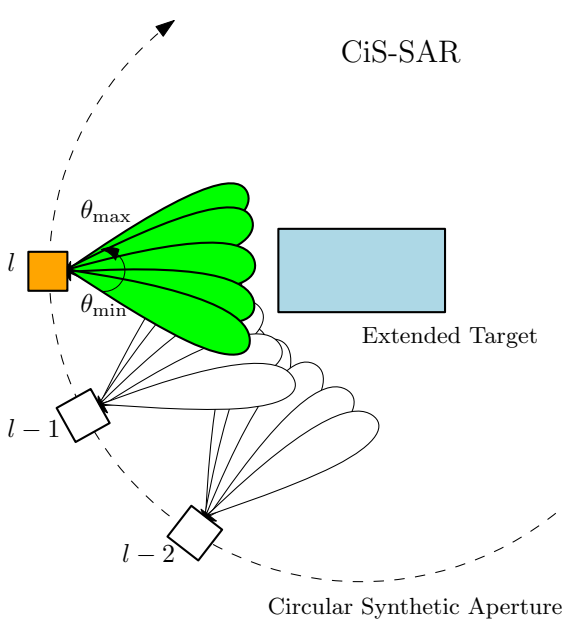

Fig. 1: SAR System Schematic

\section{System Model And Image Reconstruction}

CiS-SAR mode, essentially, combines scene scanning with SAR processing over a circular aperture. It can be thought of as the classical spotlight-mode SAR, with the difference that the target scene is scanned by the radar at each aperture position. This essentially results in a target image for each aperture sample. Figure 1 shows the schematic for CiS-SAR, where at each aperture position $l \in[1, L]$ (over the circular aperture), the radar scans the target over an angular range $\theta \in$ $\left[\theta_{\min }, \theta_{\max }\right]$ (w.r.t. radar's field of view). In general terms, a full-aperture target image would collect contributions from all the aperture positions, i.e., $l=1, \cdots, L$. However, a subaperture image would contain contributions only from a subset of the aperture positions, i.e., coherent integration is carried out over the subset. Let, for the $q$ th sub-aperture, $\mathcal{C}_{q}$ denotes the set of indices of the aperture samples of a constant-size sub-aperture, i.e., $\left|\mathcal{C}_{1}\right|=\cdots=\left|\mathcal{C}_{q}\right|=\cdots=\left|\mathcal{C}_{Q_{\mathcal{C}}}\right|$, where $Q_{\mathcal{C}}$ denotes the total number of such sub-apertures, and $\mathcal{A}_{q}$ denote the set of indices of the aperture samples of an adaptive-size sub-aperture, i.e., $\left|\mathcal{A}_{1}\right| \not \cdots \nsubseteq\left|\mathcal{A}_{q}\right| \not \cdots \not\left|\mathcal{C}_{Q_{\mathcal{A}}}\right|$, where $Q_{\mathcal{A}}$ denotes the total number of such sub-apertures, and $¥$ denotes 'not necessarily equal', i.e., the sub-apertures can have different sizes.

Here, we briefly describe image reconstruction for the CiSSAR mode. Let, at aperture position $l$ and scan angle $\theta$, the radar illuminates the target scene by a frequency modulated continuous wave (FMCW) signal with carrier frequency $f_{0}$, bandwidth $B$, pulse repetition interval (PRI) $T$ and chirp rate $\beta \triangleq B / T$, for fast time $t: 0 \leq t<T$, i.e.,

$$
s^{\operatorname{Tx}}(t)=\exp \left(j 2 \pi f_{0} t+j \pi \beta t^{2}\right) .
$$

The received signal, after dechirping, low-pass filtering and deskewing [2], can then be written as

$$
s_{l, \theta}(t)=\sum_{u=1}^{U} \alpha_{u} \exp \left(j 2 \pi\left(f_{0} \tau_{l, \theta}(u)+\beta \tau_{l, \theta}(u) t\right)\right)
$$

where $\tau_{l, \theta}(u)$ is the two-way time-delay, $\alpha_{u}$ is the reflectivity coefficient and $U$ is the total number of scatterers. The range profile of the target scene $x_{l, \theta}(r)$ can be obtained by taking a Fourier transform of $s_{l, \theta}(t)$ in (2) w.r.t., $t$, and using the linear transformation, $r=f c / 2 \beta$, where $r \in\left(0, R_{\max }\right]$ is the range variable (with $R_{\max }$ being the maximum unambiguous range) and $c$ denotes speed of light. The range resolution can be defined as, $\Delta_{r} \triangleq c / 2 B$, for $N_{r} \triangleq R_{\max } / \Delta_{r}$ range bins. Now, the target scene reflectivities along azimuth, for a given range bin $r$, can be defined as, $x_{l, r}(\theta)=\left\{x_{l, \theta}(r)\right\}_{\theta=\theta_{\min }}^{\theta_{\max }}$, i.e., collecting samples of $x_{l, \theta}(r)$, for a fixed $r$, over the scan variable $\theta$. The measured signal along the azimuth $y_{l, r}(\theta)$ can then be written as [39],

$$
y_{l, r}(\theta)=h(\theta) \star x_{l, r}(\theta)+n_{l, r}(\theta)
$$

where $h(\theta)$ is the antenna beam-pattern (uniformly sampled over beam-width $\theta \in[-\phi,+\phi]), \star$ represents the convolution operation and $n_{l, r}(\theta)$ is additive white Gaussian noise (AWGN). Let the measurements from angular scan are obtained at an angular interval $\Delta_{\theta}$. Then, $y_{l, r}(\theta)$ can be represented as an $N_{\theta} \times 1$ vector $\mathbf{y}_{l, r}$, with $N_{\theta}=\left\lfloor\left(\theta_{\max }-\right.\right.$ $\left.\left.\theta_{\min }\right) / \Delta_{\theta}\right\rfloor$, and $h(\theta)$ can be represented as an $N_{h} \times 1$ vector h, with $N_{h}=\left\lfloor 2 \phi / \delta_{\theta}\right\rfloor+1$, where $\delta_{\theta} \triangleq \Delta_{\theta} / \xi$ denotes a finer angular resolution with $\xi \geq 1$ being a constant. Similarly, $n_{l, r}(\theta)$ and $x_{l, r}(\theta)$ can be represented as $N_{\theta} \times 1$ and $N_{x} \times 1$ vectors, $\mathbf{n}_{l, r}$ and $\mathbf{x}_{l, r}$, respectively, where $N_{x}=\xi N_{\theta}+N_{h}-1$ (See [7] for more details). Thus, (3) can be written as

$$
\mathbf{y}_{l, r}=\mathbf{G H x}_{l, r}+\mathbf{n}_{l, r}
$$

where $\left(\xi N_{\theta}-1\right) \times N_{x}$ block-Toeplitz matrix $\mathbf{H}$ represens the convolution between antenna beam-pattern and the target reflectivities, and $\mathbf{G}$ is an $N_{\theta} \times\left(\xi N_{\theta}-1\right)$ selection matrix, i.e., comprising of $N_{\theta}$ rows of matrix $\mathbf{I}_{\left(\xi N_{\theta}-1\right)}$. From (4), collecting measurements for all range bins, we can write

$$
\mathbf{Y}_{l}=\mathbf{G H X}_{l}+\mathbf{N}_{l}
$$

where $\mathbf{Y}_{l}, \mathbf{X}_{l}$ and $\mathbf{N}_{l}$ are $N_{\theta} \times N_{r}, N_{x} \times N_{r}$ and $N_{\theta} \times N_{r}$ matrices, respectively.

Now, a SAR image via back-projection (BP) can be created by essentially projecting all measurements back to the target scene from all viewing positions. In the case of CiS-SAR, these viewing positions comprise both the positions w.r.t. the scanning angles as well as the positions over the aperture. For this, the measurements are upsampled via interpolation and then integrated coherently to capitalise on the SAR gains. Mathematically, image reconstruction via BP, w.r.t. point $\left(x_{i}, y_{j}\right)$, for $i=1, \cdots, I$ and $j=1, \cdots, J$, over the Cartesian grid, can be represented as

$$
\gamma_{i j}=\sum_{l} \sum_{\theta}\left[\uparrow_{\kappa, \kappa}\left(\mathbf{Y}_{l}\right)\right]_{\theta, I_{r_{i j}}}
$$

where $\uparrow_{\kappa, \kappa^{\prime}}(\cdot)$ denotes an upsampling function which interpolates a matrix by an order $\kappa$ along its rows and by an order $\kappa^{\prime}$ along its columns, $I_{r_{i j}}$ is the column index corresponding to range $r_{i j} \triangleq \sqrt{x_{i}^{2}+y_{j}^{2}}$. From (6), let

$$
\gamma_{i, j}^{l} \triangleq \sum_{\theta}\left[\uparrow_{\kappa, \kappa}\left(\mathbf{Y}_{l}\right)\right]_{\theta, I_{r_{i j}}}
$$


denotes the contribution of $l$ th aperture position to a targetscene point $\left(x_{i}, y_{j}\right)$, then, a SAR image over full-aperture via coherent integration can be described as

$$
\gamma_{i j}^{\mathrm{FA}}=\sum_{l=1}^{L} \gamma_{i, j}^{l} .
$$

Whereas, a SAR image via sub-aperture method with GLRT based image formation can be described as

$$
\gamma_{i j}^{\mathrm{SA}}=\max _{q}\left|\sum_{l \in \mathcal{C}_{q}} \gamma_{i, j}^{l}\right|
$$

where the size of set $\mathcal{C}_{q}$ is the same for all $q$. Similarly, a SAR image with adaptive sub-aperture method with GLRT based image formation can be represented as

$$
\gamma_{i j}^{\mathrm{AdSA}}=\max _{q}\left|\sum_{l \in \mathcal{A}_{q}} \gamma_{i, j}^{l}\right|
$$

where the size of set $\mathcal{A}_{q}$ is determined adaptively w.r.t. the SPA of the scatterer at point $\left(x_{i}, y_{j}\right)$. Note, the real challenge in this case is to determine the elements (and size) of $\mathcal{A}_{q}$. This paper, essentially, focuses on this aspect.

Apart from BP, an image can be reconstructed via superresolution techniques. In this respect, we opt for our recently proposed fused LASSO [37] based technique. For this, we first vectorise (5) as

$$
\mathbf{y}_{l}=\boldsymbol{\Phi}_{\mathbf{x}_{l}}+\mathbf{n}_{l}
$$

where $\mathbf{y}_{l} \triangleq \operatorname{vec}\left(\mathbf{Y}_{l}\right), \mathbf{x}_{l} \triangleq \operatorname{vec}\left(\mathbf{X}_{l}\right)$ and $\mathbf{n}_{l} \triangleq \operatorname{vec}\left(\mathbf{N}_{l}\right)$ are $N_{\theta} N_{r} \times 1, N_{x} N_{r} \times 1$ and $N_{\theta} N_{r} \times 1$ vectors, respectively, and $\mathbf{\Phi} \triangleq\left[\mathbf{I}_{N_{r}} \otimes(\mathbf{G H})\right]$ is an $N_{\theta} N_{r} \times N_{x} N_{r}$ measurement matrix. Note, since $N_{\theta} \ll N_{x}$, (11) is an under-determined system of linear equations. Assuming sparsity in $\mathbf{x}_{l}$, the optimisation problem under fused LASSO, takes the following form.

$$
\hat{\mathbf{x}}_{l}=\underset{\mathbf{x}_{l}}{\arg \min }\left\|\mathbf{y}_{l}-\mathbf{\Phi} \mathbf{x}_{l}\right\|_{2}^{2}+\lambda_{e}\left\|\mathbf{x}_{l}\right\|_{1}^{1}+\lambda_{f}\left\|\mathbf{D} \mathbf{x}_{l}\right\|_{1}^{1}
$$

where $\lambda_{e}$ and $\lambda_{f}$ are positive penalty parameters w.r.t. element-wise sparsity and fusion, respectively, and $\mathbf{D}$ is the $N_{x} N_{r} \times N_{x} N_{r}$ fusion matrix, i.e., $\mathbf{D} \mathbf{x}_{l}$ is a vector containing the differences of consecutive elements of $\mathbf{x}_{l}$ [37]. Note, (12) is convex and it can be solved via any available convex solver. In our case we use alternating direction method of multipliers (ADMM) [40], [41]. Now, the estimated $\hat{\mathbf{x}}_{l}$ can be reshaped into an $N_{x} \times N_{r}$ matrix $\hat{\mathbf{X}}_{l}$ and a reconstructed SAR image can be obtained as

$$
\gamma_{i j}=\sum_{l}\left[\uparrow_{1, \kappa}\left(\hat{\mathbf{X}}_{l}\right)\right]_{I_{\theta_{i j}}, I_{r_{i j}}}
$$

where $I_{\theta_{i j}}$ is the row index and $I_{r_{i j}}$ is the column index in $\uparrow_{1, \kappa}\left(\hat{\mathbf{X}}_{l}\right)$, corresponding to range $r_{i j}$ and angle $\theta_{i j} \triangleq$ $\arctan \left(y_{j} / x_{i}\right)$, respectively. Now, let

$$
\gamma_{i, j}^{l} \triangleq\left[\uparrow_{1, \kappa^{\prime}}\left(\hat{\mathbf{X}}_{l}\right)\right]_{I_{\theta_{i, j}}, I_{r_{i, j}}}
$$

denotes the contribution of $l$ th aperture position to point $\left(x_{i}, y_{j}\right)$, then, a SAR image via full-aperture method can be obtained via (8). Similarly, SAR images for sub-aperture and adaptive sub-aperture based methods can be obtained via (9) and (10), respectively.

\section{Change-Point Detection}

A change point is a sample within a time/data series at which some statistical property (e.g., mean or variance) of the series changes abruptly. A number of algorithms have been proposed in the literature to find change points, e.g., [32][36]. Generally, segmentation algorithms can be grouped into three categories, i.e., $i$ ) sliding window, ii) top down and iii) bottom up. In the sliding window algorithms, a segment is grown until the error of adding a new point to the segment exceeds some pre-determined threshold. Top-down algorithms work by splitting the entire trace until a pre-determined error threshold is reached. Bottom-up algorithms start by arbitrarily dividing the trace into multiple segments and then recursively merging two or more adjacent segments.

The approach used in our proposed method is the binary segmentation (BS) approach which is a very well-established top-down search method in the change-point detection literature [32]. It provides approximate results instead of exact results. Therefore, it is computationally very efficient, with a computational complexity of $\mathcal{O}(L \log L)$, where $L$ is the total number of points in the data series. Exact methods, e.g., [33], [34] can provide more accurate segmentation. However, their computational complexity can be a bottle-neck for practical implementation, especially for large datasets. Therefore, we consider BS in our proposed method to highlight the advantage of using change-point detection as a means to determine adaptive sub-apertures.

Let, $d_{1: L}=\left(d_{1}, \cdots, d_{L}\right)$, denotes a time/data series with a certain ordering. Then, the positions of $Q$ change points in the series can be denoted as, $c_{1: Q}=\left(c_{1}, \ldots, c_{Q}\right)$, where the integer $c_{q} \in[1, L-1]$, for $q=1, \cdots, Q$. Assuming, $c_{0}=0, c_{Q+1}=L$ and $c_{q}<c_{q+1}$, the $Q$ change points split the data into $Q+1$ segments. Consequently, the $q$ th segment contains $d_{\left(c_{q-1}+1\right): c_{q}}$. Generally, the detection task of identifying multiple change points can be represented as a minimisation problem of the following objective function.

$$
\sum_{q=1}^{Q+1} \mathcal{K}\left(d_{\left(c_{q-1}+1\right): c_{q}}\right)+\beta f(Q)
$$

where $\beta f(Q)$ is a penalty term (with $\beta$ being a positive constant, which can be considered as a change-point threshold) required to avoid over-fitting and $\mathcal{K}(\cdot)$ denotes a cost function. A common choice of the penalty is the linear penalty in the number of change points, i.e., $\beta f(Q)=\beta Q$. Similarly, a common choice of the cost function is the negative loglikelihood function.

The BS method begins by seeking to find a single change point. A change point $c$ is identified if it satisfies

$$
\mathcal{K}\left(d_{1: c}\right)+\mathcal{K}\left(d_{c+1: L}\right)+\beta<\mathcal{K}\left(d_{1: L}\right) .
$$

If, for a given change point $c$, (16) is not satisfied then $c$ is not the change point. Otherwise the data are split into two segments, before and after the change point. The detection step is then applied to each of the new segments. The procedure is repeated until no further change points can be detected. Note, (16) assumes that there exists a cost function parameterised 

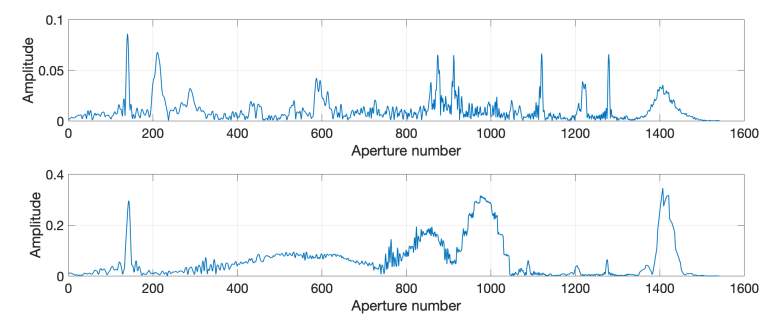

Fig. 2: Scattering responses from two pixels within the backhoe dataset.

by a certain property of the dataset for which (16) is valid. Also, from (16), we can see that BS assumes $f(Q)=1$.

The algorithm can be easily implemented via Matlab (function) libraries. Common statistics used for the cost function are mean, standard deviation (STD) and root mean square (RMS). For a data segment $d_{\mu: \nu}$, the cost function can take the following least-squares-based form to detect changes w.r.t. the mean [35].

$$
\mathcal{K}^{\text {Mean }}\left(d_{\mu: \nu}\right)=\sum_{\iota=\mu}^{\nu}\left(d_{\iota}-\left\langle d_{\mu: \nu}\right\rangle\right)^{2}
$$

where $\langle\cdot\rangle$ provides the sample mean of the data segment. To detect changes w.r.t. STD, the cost function can take the following form [35].

$$
\mathcal{K}^{\operatorname{STD}}\left(d_{\mu: \nu}\right)=\zeta_{\mu \nu} \log \left(\frac{1}{\zeta_{\mu \nu}} \sum_{\iota=\mu}^{\nu}\left(d_{\iota}-\left\langle d_{1: L}\right\rangle\right)^{2}\right)
$$

where $\zeta_{\mu \nu} \triangleq \nu-\mu+1$ is the length of data segment $d_{\mu: \nu}$. Similarly, to detect changes w.r.t. RMS, (18) is utilised while assuming $\left\langle d_{1: L}\right\rangle=0$, i.e.,

$$
\mathcal{K}^{\mathrm{RMS}}\left(d_{\mu: \nu}\right)=\zeta_{\mu \nu} \log \left(\frac{1}{\zeta_{\mu \nu}} \sum_{\iota=\mu}^{\nu} d_{\iota}^{2}\right) .
$$

\section{AdAPtive Sub-Aperture InTEgRation}

Most of the sub-aperture approaches use a constant subaperture size. This is to ensure the same resolution for each sub-aperture. However, due to difference of SPA of different scatterers over the aperture, this approach can render itself counter-purposes. If the sub-aperture size is larger than the SPA of a scatterer, then coherent integration over the subaperture, potentially, accumulates noise along with the scattering response. Similarly, if the sub-aperture size is smaller than the SPA of a scatterer, then the scattering response would spill over to the adjacent sub-aperture. Thus, image-resolution enhancement cannot be guaranteed without estimating the SPA of a scatterer.

The aspect-dependent nature of the scattering response of a scatterer can be ascertained by plotting it against the aspect angles. Figure 2 shows the scattering response of two pixels from the backhoe dataset [38] (over 1540 apertures, for a circular aperture of $110^{\circ}$, at an elevation angle of $30^{\circ}$ ). We can see that the response from both the pixels has multiple peaks and that the peaks have different widths. Therefore,
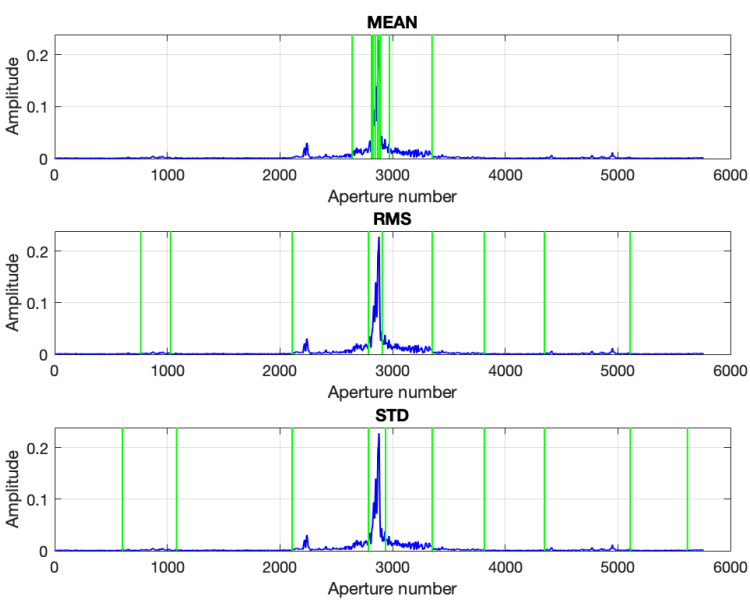

Fig. 3: Change points (green vertical lines) with 10 pre-defined change points, to detect statistical changes in the scattering response.

fixing a constant sub-aperture size would not be the best option. Instead, an adaptive sub-aperture w.r.t. the SPA of the scatterer would be a better course of action. The main question here is how to estimate the SPA of the scatterer. If the scattering response has only one peak, the SPA can be defined by locating the peak and then estimating the angular range, either by a threshold value or by fitting a Gaussian function (i.e., deriving the SPA from the fitting parameters) [29]. However, in case of multiple peaks in the scattering response, a better method is needed to take account of all the peaks and their respective spread to derive a suitable SPA for the scatterer. In this regard, we propose to use the changepoint detection method. As explained in Section III, changepoint detection is based on capturing changing statistics in different segments of the data. Thus, it provides a flexible and robust approach to identify all significant peaks in the scattering response along with their spread. In the following we explain how the method is implemented.

First a pre-defined number of change points (e.g., $10-20$ ) are used to ensure that all peaks are captured. Figure 3 shows the scattering response at a single pixel for 5600 aspect angles in a circular aperture from the dataset described in [42]. Change points (w.r.t. mean, RMS and STD, as change statistics) are denoted by vertical green lines. It can be seen, when the pre-defined change points are 10 , there is a clear response at around aperture 2300 which has not been picked up. However, when the number of pre-defined change points is increased to 20, as shown in Figure 4, the peak at aperture 2300 is recognised by the change points on either side. Thus, a slight over-estimate of the peaks in the scattering response can capture all of the significant peaks.

Using an over-estimate of scattering-response-peaks in change-point detection can cause the inclusion of nonsignificant data segments. Therefore, as a second step in our proposed method, we remove these non-significant data segments. For this, we use a threshold parameter that is learnt 

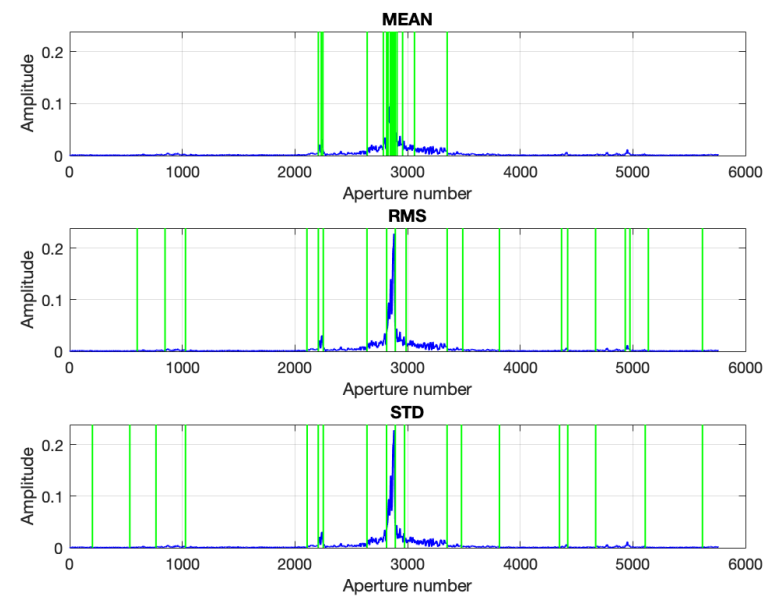

Fig. 4: Change points (green vertical lines) with 20 pre-defined change points, to detect statistical changes in the scattering response.
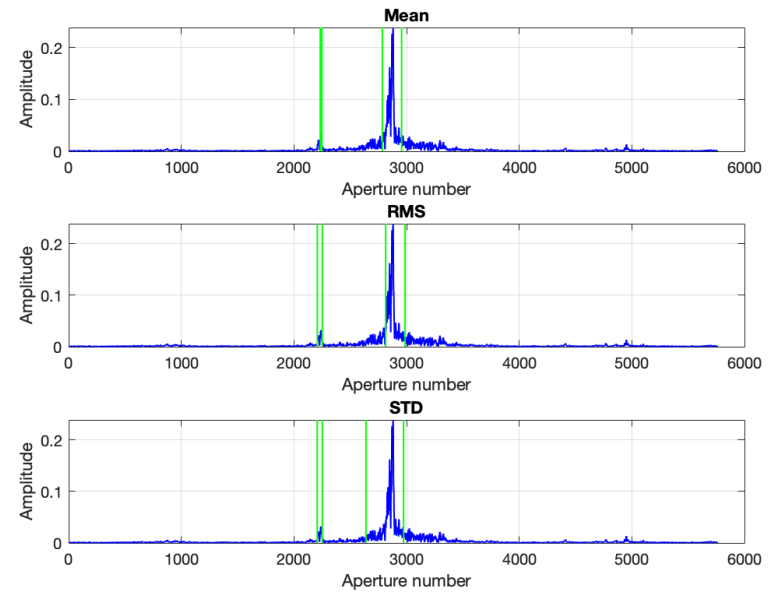

Fig. 5: Change points (green vertical lines) after noise rejection and merging the adjacent change points.

from the data itself. It can be seen that a large number of data samples in the scattering response (of the circular aperture) are noise. Thus, the mean of all the data samples is a reasonable heuristic to approximate the noise. Any data segment with mean greater than a scalar-multiple of this threshold can be defined as significant, otherwise, it can be considered as noise and discarded from subsequent processing.

As a third step, in case there are multiple adjacent change points, we merge the adjacent change points to maximise the coherent integration gain. This is an important step because it also takes care of the issue of scattering-response-peaks overestimation in the second step which may cause a bifurcation of a single peak (as can be seen in Figure 4). The final set of change points, after the last two steps, is shown in Figure 5. We can see that our proposed method has resulted in identifying all the significant peaks in the scattering response with reasonable

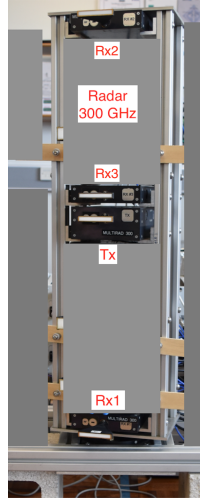

(a) Radar, $300 \mathrm{GHz}$

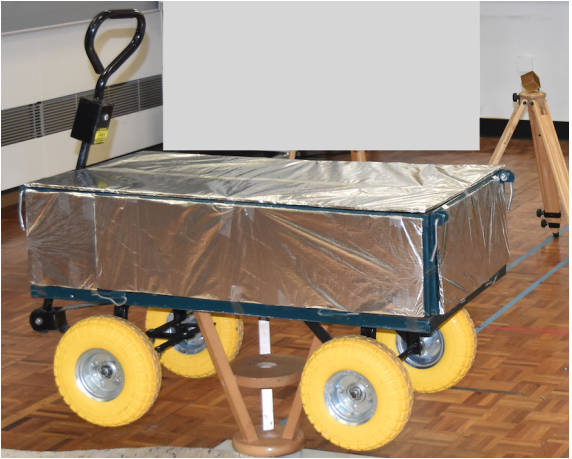

(b) Trolley on a Turn-Table
Fig. 6: Experimental Setup

TABLE I: Specifications of $300 \mathrm{GHz}$ Radar

\begin{tabular}{l|c}
\hline Modulation & FMCW \\
\hline Frequency Range & $281-299 \mathrm{GHz}$ \\
\hline Transmit Bandwidh $(B)$ & $18 \mathrm{GHz}$ \\
\hline Chirp Duration $(T)$ & $1 \mathrm{~ms}$ \\
\hline Sampling Frequency & $4.096 \mathrm{MHz}$ \\
\hline Angular Step $\left(\Delta_{\theta}\right)$ & $0.25^{\circ}$ \\
\hline Two-way $3 \mathrm{~dB}$ Beamwidth $\left(\theta_{3 \mathrm{~dB}}\right)$ & $1.3^{\circ}$ \\
\hline
\end{tabular}

noise-rejection.

\section{EXPERIMENTAL RESULTS}

In order to validate our proposed methodology, we primarily use the real data collected with a $300 \mathrm{GHz}$ radar, in our lab, for CiS-SAR. Figure 6a shows the radar. The radar consists of a single transmitter ( $\mathrm{Tx}$ ) and three vertically-aligned receivers $(\mathrm{Rx})$. We use the measurements collected from two receivers, i.e., Rx2 and Rx3. The corresponding channel $(\mathrm{CH})$ data is marked as $\mathrm{CH} 2$ and $\mathrm{CH} 3$. The radar emits FMCW signal with bandwidth of $18 \mathrm{GHz}$. Figure 7 shows the measured antenna pattern of the radar, with a two-way 3 -dB beam-width $\theta_{3 \mathrm{~dB}}=1.3^{\circ}$. Table I shows the radar specifications. For the target, we use a $1 \times 0.5 \times 0.55$ (length $\times$ width $\times$ height $\mathrm{m}^{3}$ trolley (Figure 6b). In order to realise the circular aperture, we put the trolley on a turn-table instead of moving the radar over the aperture. Each turn of the trolley emulates a sample over the circular aperture. Angular steps for the turn-table are $0.1^{\circ}$ apart, i.e., a total of $L=3600$ aperture samples for the full $360^{\circ}$ rotation. Note, for practical applications, it can be a section of the full rotation. For each aperture position, the radar scans the target scene over an angular range of $\theta= \pm 9^{\circ}$, at angular intervals $\Delta_{\theta}=0.25^{\circ}$. Figure 8 shows the measurement schematic.

We compare the performance of our proposed adaptive integration method with $i$ ) the standard full-aperture coherent integration method (8), denoted as FA, and ii) the sub-aperture integration (for a constant sub-aperture size of $2^{\circ}$ ) followed by GLRT-based composite imaging method (9), denoted as SA. For our proposed method (10), we use two statistics for 


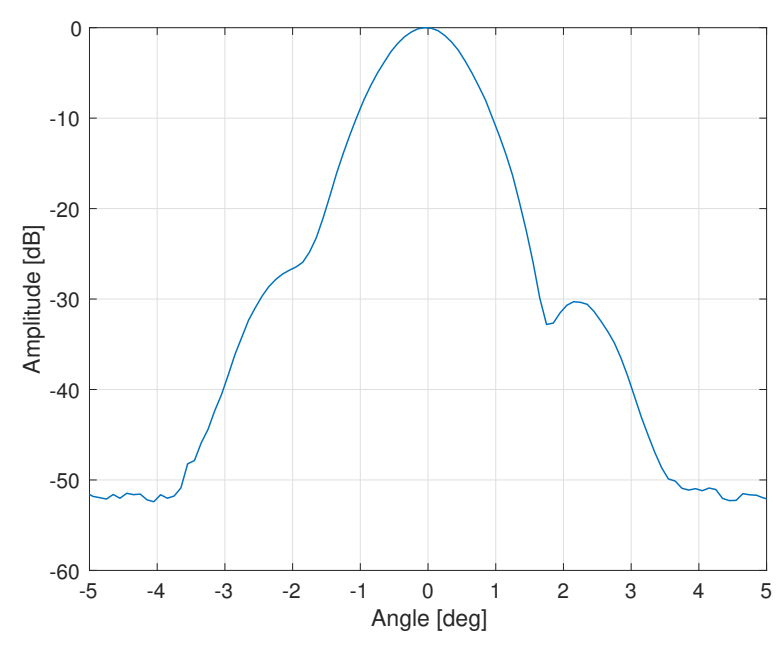

Fig. 7: Measured antenna pattern of the radar.

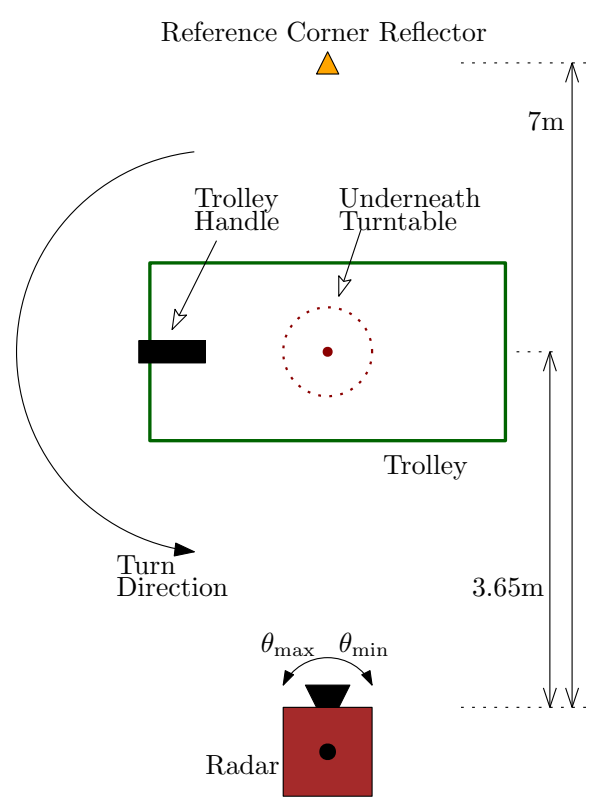

Fig. 8: Measurement Schematic

change-point detection, i.e., mean (17) and RMS (19). With the former statistics, we denote our method as AdSA-Mean and with the latter statistics, we denote our method as AdSARMS. Note, the composite image in both the cases is formed via GLRT. The number of pre-defined change points are 20 .

Figure 9 shows the performance of FA, SA, AdSA-RMS and AdSA-Mean aperture integration methods, for $\mathrm{CH} 2$ radar data, with image reconstruction via back-projection (BP). From a qualitative perspective, we can see that our proposed methods, AdSA-RMS and AdSA-Mean, show very sharp images. In contrast, the FA and SA methods show higher side-lobes, as evident by the amount of scattering around the target structure. A zoomed-in perspective of these images is provided in Figure 10, showing the right side of the trolley. It can be seen clearly that the proposed methods have better performance than the standard integration methods in terms of image resolution.

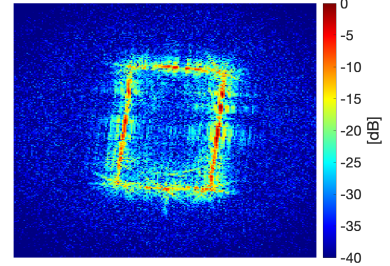

(a) FA

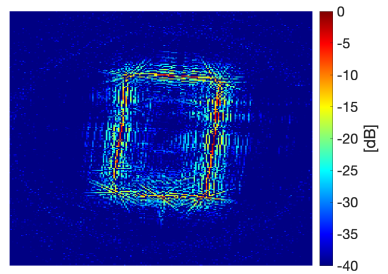

(c) AdSA-RMS

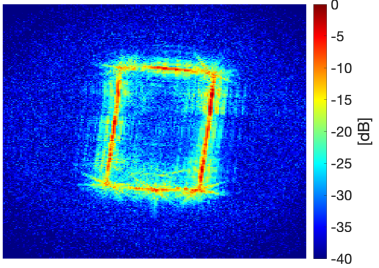

(b) SA

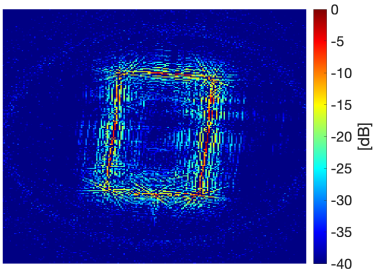

(d) AdSA-Mean
Fig. 9: Aperture Integration methods, with BP-based image reconstruction, for $\mathrm{CH} 2$.

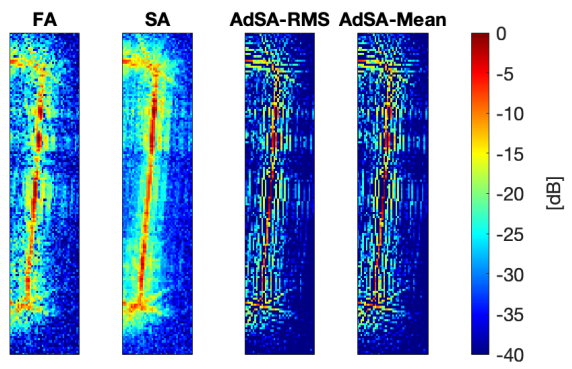

Fig. 10: Comparison of integration methods, with BP-based image reconstruction, for $\mathrm{CH} 2$.

We also provide performance results of different aperture integration methods with image reconstruction via fused LASSO (FLASSO). Figure 11 shows these imaging results of FA, SA, AdSA-RMS and AdSA-Mean methods, for $\mathrm{CH} 2$ trolley data. Again, we can observe that our proposed methods produce sharper images of the target. Figure 12 provides a zoomed-in perspective of these images, which shows that the standard integration methods result in higher side-lobe levels and thus, result in lower image resolution.

Apart from qualitative assessment, we also provide quantitative analyses of the performance of the aperture integration methods. For this, we use two performance metrics. The first performance metric is known as $3-\mathrm{dB}$ main-lobe width (MLW) [43], which provides a relative measure of spatial resolution of the radar image (in meters). We focus on the strongest scatterers [6]. This metric is calculated by finding 


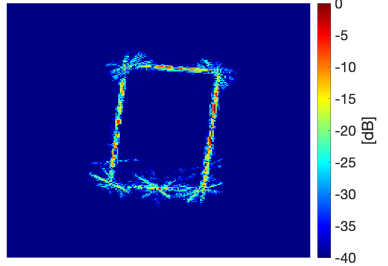

(a) FA

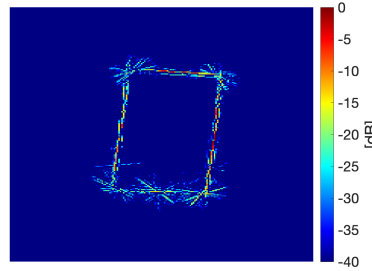

(c) AdSA-RMS

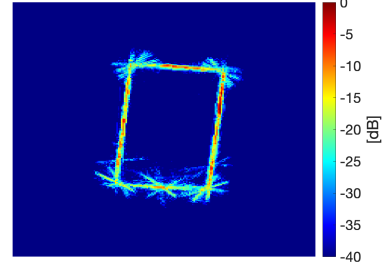

(b) SA

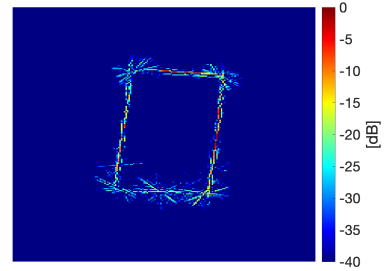

(d) AdSA-Mean
Fig. 11: Aperture Integration methods, with fused LASSObased image reconstruction, for $\mathrm{CH} 2$.

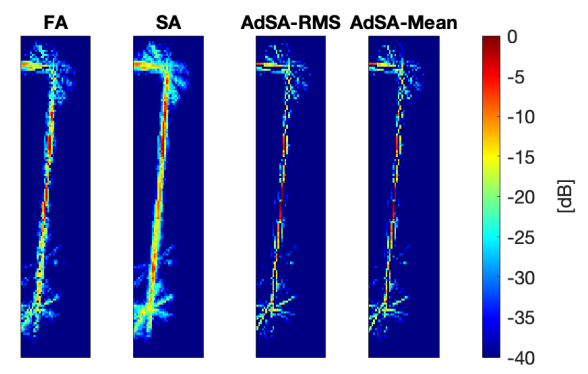

Fig. 12: Comparison of integration methods, with fused LASSO-based image reconstruction, for $\mathrm{CH} 2$.

the distance of the nearest point $3 \mathrm{~dB}$ below a strong scatterer and then averaging such distances of all of the strong scatterers (see Appendix A for a mathematical expression). Note, a finer estimate is obtained by first interpolating the in-between points. This relative measure gives a reasonable estimate of the spatial resolution of the radar image, as it directly gages the side-lobes. The lower the MLW value, the higher the spatial resolution. The second performance metric measures the speckle [43]. This parameter essentially measures variance of the smooth non-target areas of the dB-valued image, which in essence estimates speckle volume (see Appendix B for a mathematical expression). Thus, we use this metric to measure the capability of an integration method to reduce speckle. The lower this metric, the lower the speckle.

Tables II and III show the MLW results of $\mathrm{CH} 2$ and $\mathrm{CH} 3$, respectively, for image reconstruction via BP. Similarly,
TABLE II: MLW Comparison for Different Integration Methods on Trolley Dataset (CH2) via Back-Projection

\begin{tabular}{l|c|c|c|c|c}
\hline & \multicolumn{5}{|c}{ No. of Strongest Scatterers } \\
& 200 & 400 & 600 & 800 & 1000 \\
\hline FA & 0.5721 & 0.5595 & 0.5712 & 0.5777 & 0.5767 \\
\hline SA & 0.6044 & 0.5836 & 0.5743 & 0.6046 & 0.6229 \\
\hline AdSA-RMS & 0.2818 & 0.3005 & 0.3024 & 0.3087 & 0.3116 \\
\hline AdSA-Mean & 0.2661 & 0.2994 & 0.3102 & 0.3082 & 0.3115 \\
\hline
\end{tabular}

TABLE III: MLW Comparison for Different Integration Methods on Trolley Dataset (CH3) via Back-Projection

\begin{tabular}{l|c|c|c|c|c}
\hline & \multicolumn{5}{|c}{ No. of Strongest Scatterers } \\
& 200 & 400 & 600 & 800 & 1000 \\
\hline FA & 0.5798 & 0.5670 & 0.5661 & 0.5824 & 0.5931 \\
\hline SA & 0.6215 & 0.6108 & 0.6235 & 0.6327 & 0.6502 \\
\hline AdSA-RMS & 0.3357 & 0.3295 & 0.3289 & 0.3335 & 0.3338 \\
\hline AdSA-Mean & 0.3130 & 0.3136 & 0.3136 & 0.3154 & 0.3184 \\
\hline
\end{tabular}

TABLE IV: MLW Comparison for Different Integration Methods on Trolley Dataset $(\mathrm{CH} 2)$ via Fused LASSO

\begin{tabular}{l|c|c|c|c|c}
\hline & \multicolumn{5}{|c}{ No. of Strongest Scatterers } \\
& 200 & 400 & 600 & 800 & 1000 \\
\hline FA & 0.4888 & 0.4789 & 0.4792 & 0.4842 & 0.4844 \\
\hline SA & 0.5052 & 0.5055 & 0.5126 & 0.5273 & 0.5325 \\
\hline AdSA-RMS & 0.2937 & 0.2955 & 0.2970 & 0.3020 & 0.3007 \\
\hline AdSA-Mean & 0.2949 & 0.2993 & 0.2958 & 0.2957 & 0.2908 \\
\hline
\end{tabular}

TABLE V: MLW Comparison for Different Integration Methods on Trolley Dataset (CH3) via Fused LASSO

\begin{tabular}{l|c|c|c|c|c}
\hline & \multicolumn{5}{|c}{ No. of Strongest Scatterers } \\
& 200 & 400 & 600 & 800 & 1000 \\
\hline FA & 0.4710 & 0.5016 & 0.4962 & 0.4996 & 0.5028 \\
\hline SA & 0.5319 & 0.5310 & 0.5279 & 0.5413 & 0.5570 \\
\hline AdSA-RMS & 0.3577 & 0.3507 & 0.3322 & 0.3295 & 0.3259 \\
\hline AdSA-Mean & 0.3288 & 0.3486 & 0.3248 & 0.3239 & 0.3266 \\
\hline
\end{tabular}

Tables IV and V, show these results for image reconstruction via FLASSO. We provide these results for varying number of strong scatterers. We can see that our proposed AdSA methods, i.e., AdSA-RMS and AdSA-Mean, outperform both the FA and SA integration methods, as they show lower values of MLW (almost by a factor of 2) for different number of scatterers. For speckle results, we focus on four smooth non-target regions of the target, as depicted in Figure 13. Tables VI-IX show these results for the four regions. The results consider both $\mathrm{CH} 2$ and $\mathrm{CH} 3$ data images when the reconstruction method is $\mathrm{BP}$ and FLASSO. We can see that in all the cases, our proposed methods display higher speckle reduction (almost by a factor of 5 or higher) than the other methods. These results validate the efficacy of our proposed methods. Note, an interesting general observation can be made about our proposed methods. We see that for MLW results, AdSA-Mean outperforms the AdSA-RMS, and for speckle 


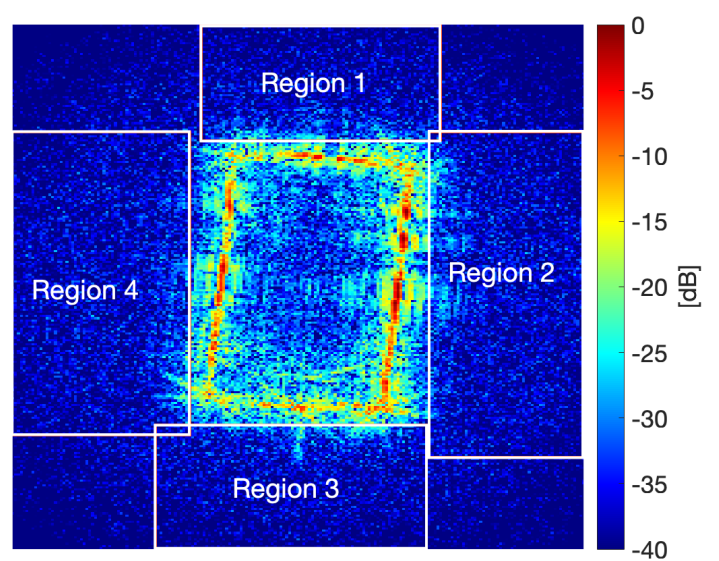

Fig. 13: Speckle Regions

results, it is vice versa. Since AdSA-Mean uses the segment mean as the change statistic, it seems this has a positive impact on reducing the side-lobes and enhancing the MLW. In case of AdSA-RMS, since RMS is used as the change statistic, which is related to data variance, it is understandable that it would have a positive impact on speckle reduction which also relates to data variance (as explained in the previous paragraph). Thus, our proposed methods can also provide the flexibility of favouring a particular performance metric, if so desired.

We also compare the performance of our proposed methods with another recently proposed adaptive integration method, i.e., [29]. Here, we refer to this method as, AdSA-[29]. As explained in Section I, this method estimates the SPA of a scatterer by fitting a Gaussian function to the scattering response, followed by determining a common SPA for all the scatterers. For illustration, we only consider $\mathrm{CH} 2$ data of the radar when the image is reconstructed via BP. Figure 14 shows this comparison. We can see that AdSA-[29] provides reasonable imaging results, however, the noise floor seems to be much higher than our imaging results. Table $\mathrm{X}$ provides the quantitive results for this comparison for MLW (750 scattterers) and speckle metrics. We can see that our proposed methods outperform AdSA-[29] for both the performance metrics.

In order to prove that our proposed methods are not bound to one dataset, we also provide the performance results for the publicly available dataset of a backhoe target [38]. This is a synthetic dataset, generated over a dome/circular aperture around the target (Figure 15) at an elevation angle of $30^{\circ}$, for the angular range $-10^{\circ}$ to $100^{\circ}$, with a bandwidth of 5.9 $\mathrm{GHz}$ and with a central frequency of $10 \mathrm{GHz}$. Figure 16 shows the aperture integration performance of FA, SA, AdSA-Mean and AdSA-RMS methods, for BP based image reconstruction. We can see that our proposed methods show sharp images with reduced noise levels. Figure 17 shows a close-up on the lower left side of the target. We can clearly see that our proposed methods have succeeded in removing the noiselike artefact in comparison to FA and SA. Table XI provides the quantitative results of this comparison for MLW metric
TABLE VI: Speckle Comparison for Different Integration Methods on Trolley Dataset (Region-1)

\begin{tabular}{l|c|c|c|c}
\hline & FA & SA & AdSA-Mean & AdSA-RMS \\
\hline BP $(\mathrm{CH} 2)$ & 14.7837 & 14.2578 & 7.2674 & 5.5332 \\
\hline BP $(\mathrm{CH} 3)$ & 14.9768 & 6.9173 & 11.9831 & 10.0790 \\
\hline FLASSO $(\mathrm{CH} 2)$ & 1.3969 & 3.1302 & 0.7906 & 0.7935 \\
\hline FLASSO $(\mathrm{CH} 3)$ & 1.9614 & 2.8420 & 1.6522 & 1.8710 \\
\hline
\end{tabular}

TABLE VII: Speckle Comparison for Different Integration Methods on Trolley Dataset (Region-2)

\begin{tabular}{l|c|c|c|c}
\hline & FA & SA & AdSA-Mean & AdSA-RMS \\
\hline BP $(\mathrm{CH} 2)$ & 13.3060 & 10.9294 & 4.8563 & 4.0300 \\
\hline BP $(\mathrm{CH} 3)$ & 10.9760 & 3.2073 & 9.4528 & 8.3557 \\
\hline FLASSO $(\mathrm{CH} 2)$ & 0.2439 & 0.2093 & 0.2080 & 0.1721 \\
\hline FLASSO $(\mathrm{CH} 3)$ & 1.0976 & 0.6558 & 0.8820 & 0.9739 \\
\hline
\end{tabular}

TABLE VIII: Speckle Comparison for Different Integration Methods on Trolley Dataset (Region-3)

\begin{tabular}{l|c|c|c|c}
\hline & FA & SA & AdSA-Mean & AdSA-RMS \\
\hline BP $(\mathrm{CH} 2)$ & 14.3353 & 22.1857 & 6.2335 & 4.7359 \\
\hline BP $(\mathrm{CH} 3)$ & 11.9102 & 20.0988 & 9.2830 & 7.9054 \\
\hline FLASSO $(\mathrm{CH} 2)$ & 1.7476 & 6.3576 & 1.0427 & 1.0689 \\
\hline FLASSO $(\mathrm{CH} 3)$ & 2.0534 & 6.3834 & 1.4258 & 1.5926 \\
\hline
\end{tabular}

TABLE IX: Speckle Comparison for Different Integration Methods on Trolley Dataset (Region-4)

\begin{tabular}{l|c|c|c|c}
\hline & FA & SA & AdSA-Mean & AdSA-RMS \\
\hline BP $(\mathrm{CH} 2)$ & 9.4450 & 16.1770 & 2.6328 & 2.0519 \\
\hline BP $(\mathrm{CH} 3)$ & 4.0698 & 5.6039 & 2.8655 & 2.4517 \\
\hline FLASSO (CH2) & 0.5490 & 1.7409 & 0.2571 & 0.2683 \\
\hline FLASSO (CH3) & 0.6251 & 1.7256 & 0.4840 & 0.5017 \\
\hline
\end{tabular}

TABLE X: Comparison for Adaptive Integration Methods on Trolley Dataset $(\mathrm{CH} 2)$ via Back-Projection

\begin{tabular}{l|c|c|c|c|c}
\hline & MLW & \multicolumn{4}{|c}{ Speckle Regions } \\
& $(750)$ & Region-1 & Region-2 & Region-3 & Region-4 \\
\hline AdSA-[29] & 0.4524 & 12.0242 & 14.5445 & 12.4758 & 9.3170 \\
\hline AdSA-RMS & 0.3080 & 5.5332 & 4.0300 & 4.7359 & 2.0519 \\
\hline AdSA-Mean & 0.3053 & 7.2674 & 4.8563 & 6.2335 & 2.63284 \\
\hline
\end{tabular}

with varying number of strong scatterers. Again, we can see that our proposed methods have outperformed the traditional approaches. Note, since this is a synthetic dataset, there is no real speckle in the data. Therefore, we have not provided speckle results. However, as mentioned earlier, regarding Figure 17, our proposed methods have shown higher noise rejection capability than the other methods.

\section{CONCLusions}

In this paper, we have presented an adaptive sub-aperture integration method for wide-angle SAR for improved imaging. We have proposed to employ change-point detection methods, 


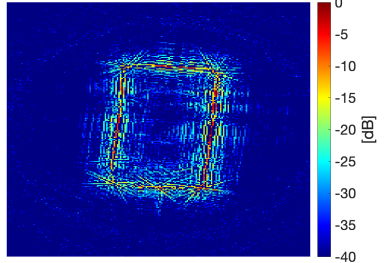

(a) AdSA-RMS

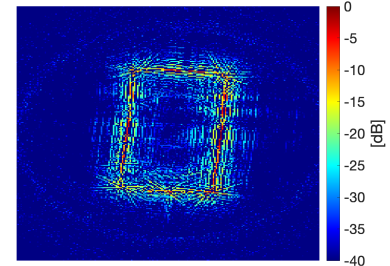

(b) AdSA-Mean

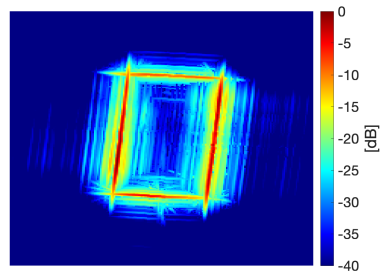

(c) AdSA-[29]
Fig. 14: Adaptive Integration methods, with BP-based image reconstruction, for $\mathrm{CH} 2$.

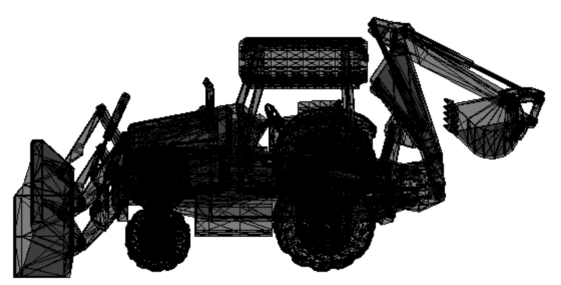

Fig. 15: Backhoe Target

which use change statistics of the scattering response data, to estimate the persistence angle of the scatterers, and consequently enhance the coherent integration gains. We have provided qualitative and quantitative analyses to gauge the performance of our proposed methods. We have compared our proposed methods with the standard integration approaches as well as a recently proposed adaptive integration approach. The performance results show that our proposed methods have the ability to enhance image resolution as well as reduce speckle/noise artefacts in comparison to the existing methods. We have presented experimental results on the real-dataset collected on our $300 \mathrm{GHz}$ radar as well as on the publicly available backhoe dataset. These results validate our claims.

TABLE XI: MLW Comparison for Different Integration Methods on Backhoe Dataset via Back-Projection

\begin{tabular}{l|c|c|c}
\hline & \multicolumn{3}{|c}{ No. of Strongest Scatterers } \\
& 1000 & 2000 & 3000 \\
\hline FA & 0.55095 & 0.57869 & 0.60043 \\
\hline SA & 0.57147 & 0.62265 & 0.68141 \\
\hline AdSA-RMS & 0.52221 & 0.54984 & 0.57218 \\
\hline AdSA-Mean & 0.4892 & 0.51167 & 0.53917 \\
\hline
\end{tabular}

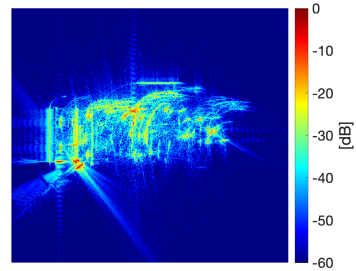

(a) FA

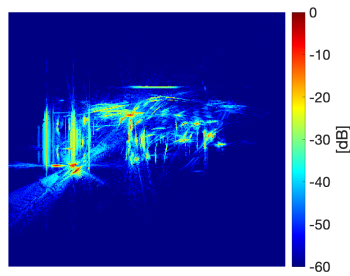

(c) AdSA-RMS

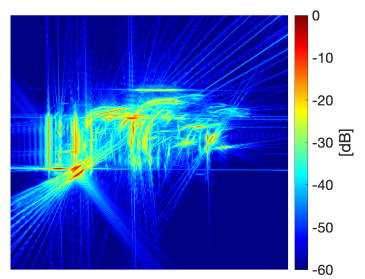

(b) SA

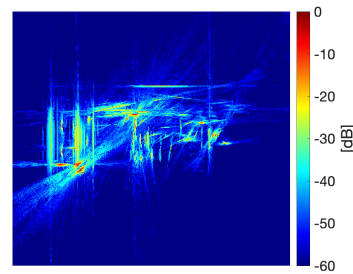

(d) AdSA-Mean
Fig. 16: Aperture Integration methods, with BP-based image reconstruction.
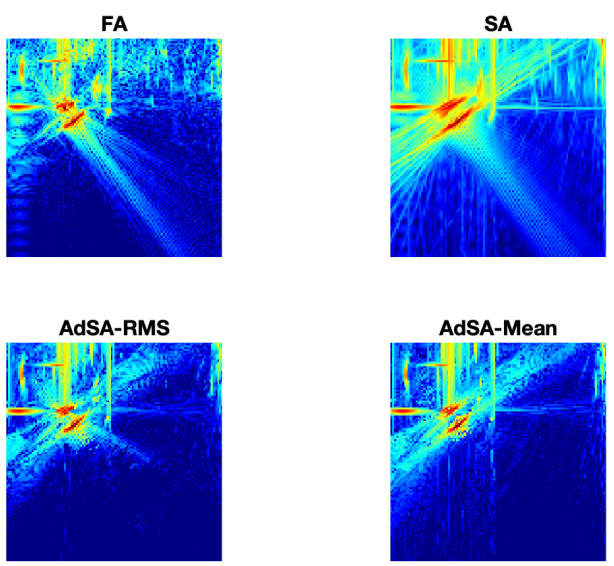

Fig. 17: Comparison of integration methods, with BP-based image reconstruction.

\section{APPENDIX A}

\section{Measure of Spatial Resolution}

As explained in Section $\mathrm{V}$, we use the 3-dB main-lobe width (MLW), as a metric to measure the spatial resolution of the radar image. The following procedure is used to find MLW. For an $I \times J$ target image, with pixel locations $\left(x_{i}, y_{j}\right)$, for $i=1, \cdots, I$ and $j=1, \cdots, J$, and respective pixel intensities, $\gamma_{i, j}$, let the brightest scatterers among these pixels are represented as $\left(x_{z}, y_{z}\right)$, for $z=1, \cdots, Z$, where $Z \subset I \times J$, with respective pixel intensities $\gamma_{z}$. Note, here we assume the pixel intensities to be in dBs. Then, the MLW for $z$ th scatterer can be obtained as

$$
\begin{aligned}
& \mathrm{MLW}_{z}= \min \sqrt{\left(x_{i}-x_{z}\right)^{2}+\left(y_{j}-y_{z}\right)^{2}}, \forall\left(x_{i}, y_{j}\right) \\
& \text { s.t. } \gamma_{i, j} \leq \gamma_{z}-3
\end{aligned}
$$


and the MLW for the whole image is obtained as

$$
\mathrm{MLW}=\sum_{z=1}^{Z} \mathrm{MLW}_{z} .
$$

Note, for a finer estimate, we interpolate over $\left(x_{i}, y_{j}\right)$ in (20a) so that (20b) is close to equality.

\section{APPENDIX B}

\section{MEASURE OF SPECKLE}

Given the pixel intensities, $\gamma_{i, j}$ in $\mathrm{dBs}$, for $i=1, \cdots, I^{\prime}$ and $j=1, \cdots, J^{\prime}$, of a smooth and non-target, $I^{\prime} \times J^{\prime}$ patch of the image, where $I^{\prime} \subset I$ and $J^{\prime} \subset J$, the speckle volume can be measured as

$$
\operatorname{var}\left(\gamma_{1,1}, \cdots, \gamma_{I^{\prime}, 1}, \cdots, \gamma_{I^{\prime}, J}\right)
$$

where $\operatorname{var}(\cdot)$ denotes the standard mathematical function of variance.

\section{REFERENCES}

[1] D. Wright, S. Gishkori, L. Daniel, M. Gashinova, and B. Mulgrew, "Adaptive integration time in automotive SAR," in 2020 IEEE Radar Conference (RadarConf20), 2020, pp. 1-6.

[2] W. Carrara, R. Goodman, and R. Majewski, Spotlight Synthetic Aperture Radar. Boston: Artech House, 1995.

[3] C. Jakowatz, D. Wahl, P. Eichel, D. Ghiglia, and P. Thompson, SpotlightMode Synthetic Aperture Radar: A Signal Processing Approach. MA, USA: Kulwer Academic Publishers, 1996.

[4] M. Soumekh, Synthetic Aperture Radar Signal Processing with MATLAB Algorithms. NY, USA: John Wiley \& Sons, Inc., 1999.

[5] M. Cetin, I. Stojanovic, O. Onhon, K. Varshney, S. Samadi, W. C. Karl, and A. S. Willsky, "Sparsity-driven synthetic aperture radar imaging: Reconstruction, autofocusing, moving targets, and compressed sensing," IEEE Signal Processing Magazine, vol. 31, no. 4, pp. 27-40, July 2014.

[6] S. Gishkori and B. Mulgrew, "Graph signal processing-based imaging for synthetic aperture radar," IEEE Geoscience and Remote Sensing Letters, vol. 17, no. 2, pp. 232-236, 2019.

[7] S. Gishkori, L. Daniel, M. Gashinova, and B. Mulgrew, "Imaging for a forward scanning automotive synthetic aperture radar," IEEE Transactions on Aerospace and Electronic Systems, vol. 55, no. 3, pp. 1420-1434, June 2019.

[8] S. Gishkori, D. Wright, L. Daniel, M. Gashinova, and B. Mulgrew, "Imaging moving targets for a forward-scanning automotive SAR," IEEE Transactions on Aerospace and Electronic Systems, vol. 56, no. 2, pp. 1106-1119, 2020.

[9] F. Friederich, W. von Spiegel, M. Bauer, F. Meng, M. D. Thomson, S. Boppel, A. Lisauskas, B. Hils, V. Krozer, A. Keil, T. Loffler, R. Henneberger, A. K. Huhn, G. Spickermann, P. H. Bolivar, and H. G. Roskos, "THz active imaging systems with real-time capabilities," IEEE Transactions on Terahertz Science and Technology, vol. 1, no. 1, pp. 183-200, 2011.

[10] K. B. Cooper, R. J. Dengler, N. Llombart, B. Thomas, G. Chattopadhyay, and P. H. Siegel, "THz imaging radar for standoff personnel screening," IEEE Transactions on Terahertz Science and Technology, vol. 1, no. 1, pp. 169-182, 2011.

[11] J. Ding, M. Kahl, O. Loffeld, and P. H. Bolvar, "THz 3-D image formation using SAR techniques: Simulation, processing and experimental results," IEEE Transactions on Terahertz Science and Technology, vol. 3, no. 5, pp. 606-616, 2013

[12] D. Wright, L. Daniel, S. Gishkori, M. Gashinova, and B. Mulgrew, "Circular imaging and phase error correction of $300 \mathrm{GHz}$ radar data," in 2019 International Radar Conference (RADAR), 2019, pp. 1-4.

[13] S. Gishkori, D. Wright, L. Daniel, M. Gashinova, and B. Mulgrew, "Improved SAR imaging via cross-learning from camera images," IEEE Transactions on Aerospace and Electronic Systems, p. to appear, 2021.

[14] R. Moses, L. Potter, and M. Cetin, "Wide-angle SAR imaging," Proc.SPIE, vol. 5427, pp. 164-175, 2004.

[15] R. L. Moses and L. C. Potter, "Noncoherent 2D and 3D SAR reconstruction from wide-angle measurements," in 13th annual adaptive sensor array processing workshop, MIT Lincoln laboratory, 2005.
[16] L. J. Moore and L. C. Potter, "Three-dimensional resolution for circular synthetic aperture radar," in Algorithms for Synthetic Aperture Radar Imagery XIV, vol. 6568 . SPIE, 2007, pp. $30-41$.

[17] L. C. Trintinalia, R. Bhalla, and Hao Ling, "Scattering center parameterization of wide-angle backscattered data using adaptive Gaussian representation," IEEE Transactions on Antennas and Propagation, vol. 45, no. 11, pp. 1664-1668, 1997.

[18] I. Stojanovic, M. Cetin, and W. C. Karl, "Joint space aspect reconstruction of wide-angle SAR exploiting sparsity," in Algorithms for Synthetic Aperture Radar Imagery XV, E. G. Zelnio and F. D. Garber, Eds., vol. 6970, International Society for Optics and Photonics. SPIE, 2008, pp. $37-48$.

[19] J. A. Jackson, B. D. Rigling, and R. L. Moses, "Canonical scattering feature models for 3D and bistatic SAR," IEEE Transactions on Aerospace and Electronic Systems, vol. 46, no. 2, pp. 525-541, April 2010.

[20] J. Ash, E. Ertin, L. C. Potter, and E. Zelnio, "Wide-angle synthetic aperture radar imaging: Models and algorithms for anisotropic scattering," IEEE Signal Processing Magazine, vol. 31, no. 4, pp. 16-26, July 2014.

[21] M. Cetin, W. C. Karl, and D. A. Castanon, "Feature enhancement and ATR performance using nonquadratic optimization-based SAR imaging," IEEE Transactions on Aerospace and Electronic Systems, vol. 39, no. 4, pp. 1375-1395, Oct 2003.

[22] L. C. Potter, E. Ertin, J. T. Parker, and M. Cetin, "Sparsity and compressed sensing in radar imaging," Proceedings of the IEEE, vol. 98, no. 6, pp. 1006-1020, June 2010.

[23] R. L. Moses and J. N. Ash, "An autoregressive formulation for SAR backprojection imaging," IEEE Transactions on Aerospace and Electronic Systems, vol. 47, no. 4, pp. 2860-2873, 2011.

[24] E. Ertin, C. D. Austin, S. Sharma, R. L. Moses, and L. C. Potter, "GOTCHA experience report: three-dimensional SAR imaging with complete circular apertures," in Algorithms for Synthetic Aperture Radar Imagery XIV, E. G. Zelnio and F. D. Garber, Eds., vol. 6568, International Society for Optics and Photonics. SPIE, 2007, pp. $9-20$.

[25] R. Hu, R. Min, and Y. Pi, "A video-SAR imaging technique for aspectdependent scattering in wide angle," IEEE Sensors Journal, vol. 17, pp. 3677-3688, 2017.

[26] R. D. Chaney, A. S. Willsky, and L. M. Novak, "Coherent aspectdependent SAR image formation," in Algorithms for Synthetic Aperture Radar Imagery, vol. 2230. SPIE, 1994, pp. 256 - 274.

[27] E. Ertin, R. L. Moses, and L. C. Potter, "Interferometric methods for three-dimensional target reconstruction with multipass circular SAR," IET Radar, Sonar Navigation, vol. 4, no. 3, pp. 464-473, 2010.

[28] Z. Yue, L. Yun, H. Wen, S. Wenjie, and X. Feiteng, "Target aspect feature extraction and application from multi-aspect high resolution SAR," in IGARSS, July 2017, pp. 1784-1787.

[29] T. Liu, Y. Pi, and X. Yang, "Wide-angle CSAR imaging based on the adaptive subaperture partition method in the terahertz band," IEEE Transactions on Terahertz Science and Technology, vol. 8, no. 2, pp. 165-173, March 2018.

[30] C. Chen and H. C. Andrews, "Target-motion-induced radar imaging," IEEE Transactions on Aerospace and Electronic Systems, vol. AES-16, no. 1, pp. 2-14, 1980.

[31] R. P. Perry, R. C. DiPietro, and R. L. Fante, "SAR imaging of moving targets," IEEE Transactions on Aerospace and Electronic Systems, vol. 35, no. 1, pp. 188-200, 1999.

[32] A. J. Scott and M. Knott, "A cluster analysis method for grouping means in the analysis of variance," Biometrics, vol. 30, no. 3, pp. 507-512, 1974.

[33] I. E. Auger and C. E. Lawrence, "Algorithms for the optimal identification of segment neighborhoods," Bulletin of Mathematical Biology, vol. 51, no. 1, pp. 39-54, 1989.

[34] B. Jackson, J. D. Scargle, D. Barnes, S. Arabhi, A. Alt, P. Gioumousis, E. Gwin, P. Sangtrakulcharoen, L. Tan, and Tun Tao Tsai, "An algorithm for optimal partitioning of data on an interval," IEEE Signal Processing Letters, vol. 12, no. 2, pp. 105-108, 2005.

[35] M. Lavielle, "Using penalized contrasts for the change-point problem," Signal Process., vol. 85, no. 8, pp. 1501-1510, Aug. 2005.

[36] R. Killick, P. Fearnhead, and I. A. Eckley, "Optimal detection of changepoints with a linear computational cost," Journal of the American Statistical Association, vol. 107, no. 500, pp. 1590-1598, 2012.

[37] R. Tibshirani, M. Saunders, S. Rosset, J. Zhu, and K. Knight, "Sparsity and smoothness via the fused LASSO," Journal of the Royal Statistical Society Series B, pp. 91-108, 2005.

[38] K. Naidu and L. Lin, "Data dome: full k-space sampling data for highfrequency radar research," Proc.SPIE, vol. 5427, pp. 200 - 207, 2004.

[39] M. A. Richards, Fundamentals of Radar Signal Processing. Two Penn Plaza, NY, USA: McGraw-Hill Companies, Inc., 2005. 
[40] D. P. Bertsekas and J. N. Tsitsiklis, Parallel and Distributed Computation: Numerical Methods. Mass.: Athena Scientific, 1997.

[41] S. Gishkori and G. Leus, "Compressed sensing for block-sparse smooth signals," in IEEE International Conference on Acoustics, Speech and Signal Processing (ICASSP), May 2014, pp. 4166-4170.

[42] K. E. Dungan, C. Austin, J. Nehrbass, and L. C. Potter, "Civilian vehicle radar data domes," in Algorithms for Synthetic Aperture Radar Imagery $X V I I$, vol. 7699. SPIE, 2010, pp. $242-253$.

[43] G. R. Benitz, "High-definition vector imaging," in Lincoln Laboratory Journal, vol. 10, no. 2, 1997, pp. 147-170.

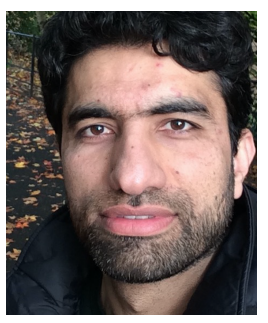

Shahzad Gishkori received the B.Sc. degree in electrical engineering from the University of Engineering and Technology Lahore, Pakistan, in 2002, and the M.Sc. (cum Laude) and Ph.D. degrees, both in electrical engineering, from the Delft University of Technology, The Netherlands, in 2009 and 2014, respectively.

From 2014 to 2015, he was a Research Associate with the Imperial College London, U.K. From 2016 to 2020, he was a Research Associate with the University of Edinburgh, U.K. Since March 2020, he is working as a Research Fellow at King Abdullah University of Science and Technology, Saudi Arabia. His research interests include signal \& image processing and machine learning.

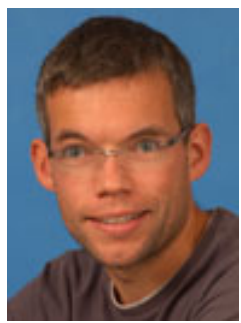

David Wright received a BSc. in Geophysics in 1998 from the University of Edinburgh, MSc. in applied Geophysics from the University of Durham in 1999 and $\mathrm{PhD}$. in exploration Geophysics from the University of Edinburgh in 2004.

$\mathrm{He}$ was co-founder and senior geophysicist at MTEM Ltd. from 2004-2007 and senior research geophysicist at Petroleum Geo Services from 20072010. From $2010-2016$ he was a research fellow in the School of Geosciences at the University of Edinburgh. Since January 2018 he has been a research associate at the institute of digital communications at the University of Edinburgh working on radar imaging for autonomous vehicles.

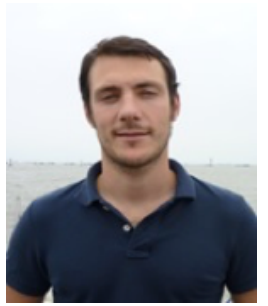

Liam Daniel received the M.Sc. degree in theoretical physics in 2005 and the Ph.D. degree in maritime forward scatter radar development in 2017, both from the University of Birmingham, Birmingham, U.K.

He is currently a Research Fellow at the Microwave Integrated Systems Laboratory, School of Engineering, University of Birmingham, Birmingham, U.K. His research interests include experimental, theoretical, simulation and signal processing aspects of bistatic radars with a focus on forward scatter radar and passive sensing, automotive sensing, and low-THz radar systems.

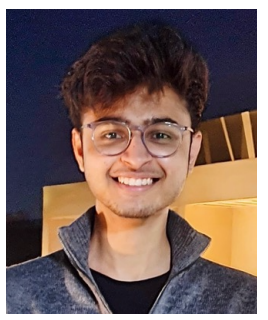

Aasim Shaikh received the B. Tech degree with Honours in electrical engineering from The Indian Institute of Technology Gandhinagar, Gujarat, India in 2021.

He is currently an MS student at King Abdullah University of Science and Technology, Thuwal, Saudi Arabia, in the Computer, Electrical and Mathematical Science and Engineering Division. His research interests include synthetic aperture radar and signal and image processing

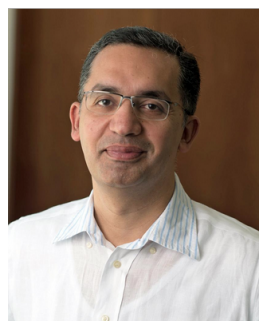

Tareq Al-Naffouri received the B.S. degrees in mathematics and electrical engineering (with first honors) from King Fahd University of Petroleum and Minerals, Dhahran, Saudi Arabia, the M.S. degree in electrical engineering from the Georgia Institute of Technology, Atlanta, in 1998, and the Ph.D. degree in electrical engineering from Stanford University, Stanford, CA, in 2004.

He was a visiting scholar at California Institute of Technology, Pasadena, CA in 2005 and summer 2006. He was a Fulbright scholar at the University of Southern California in 2008. He is currently a Professor at the Electrical Engineering Department, King Abdullah University of Science and Technology (KAUST). His research interests lie in the areas of sparse, adaptive, and statistical signal processing and their applications to wireless communications and localization, machine learning, and network information theory. He has over 300 publications in journal and conference proceedings and 20 issued/pending patents.

Dr. Al-Naffouri is the recipient of the IEEE Education Society Chapter Achievement Award in 2008 and Al-Marai Award for innovative research in communication in 2009. Dr. Al-Naffouri was an Associate Editor of IEEE Transactions on Signal Processing 2013-2018.

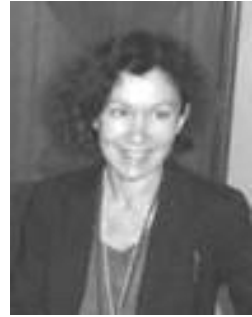

Marina Gashinova received the M.Math degree from St.-Petersburg State University, Saint Peterburg, Russia, in 1991, and the Ph.D. degree in physics and mathematics from St.-Petersburg Electrotechnical University, Saint Petersburg, Russia, in 2003.

In 2006, she joined the Microwave Integrated System Laboratory, University of Birmingham, Birmingham, U.K., as a Research Fellow. She is currently a Professor of Radar and RF Sensors, leading the research group on passive and active bistatic radar, $\mathrm{THz}$ multi-feature imaging radar, and automotive sensors.

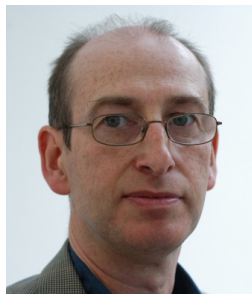

Bernard Mulgrew (FIEEE, FREng, FRSE, FIET) received the B.Sc. degree from Queen's University Belfast, Belfast, U.K., in 1979, and the Ph.D. degree from University of Edinburgh, Edinburgh, U.K., in 1987.

After graduation, he worked for 4 years as a Development Engineer with the Radar Systems Department, Ferranti, Edinburgh. From 1983 to 1986, he was a Research Associate with the Department of Electrical Engineering, University of Edinburgh, Edinburgh, U.K. He was appointed as a Lecturer in 1986, promoted to a Senior Lecturer in 1994 and became a Reader in 1996. The University of Edinburgh appointed him to a Personal Chair in October 1999 (Professor of Signals and Systems). He has co-authored three books on signal processing. His research interests include adaptive signal processing and estimation theory and in their application to radar and sensor systems. 